\title{
Color Control System for RGB LED Light Sources Using Junction Temperature Measurement
}

\author{
Xiaohui Qu, Siu Chung Wong and Chi K. Tse \\ Department of Electronic and Information Engineering, The Hong Kong Polytechnic University, Hong Kong
}

\begin{abstract}
The efficiency of LED lights is approaching that of fluorescent lamps. LED light sources are finding more applications than conventional light bulbs due to their compactness, lower heat dissipation, and most importantly, real-time color changing capability. Accurate control of colors for RGB LED lights is a challenging task, which includes optical color mixing, color light intensity control and color point maintenance due to LED junction temperature change and device aging. In this paper , we present a LED junction temperature measurement technique for a pulse width modulation (PWM) diode forward current controlled RGB LED lighting system. The technique can control the color effectively without the need for using expensive feedback systems involving light sensors. Performance of chromaticity and luminance stability for a temperature compensated RGB LED system will be presented.
\end{abstract}

Index Terms-RGB LED, color control, temperature compensation, LED lighting.

\section{INTRODUCTION}

Light-emitting-diode (LED) lightings are expected to become a major kind of light sources for the coming decades [1]. Red-green-blue (RGB) LEDs having capability of generating instantly different colors can find many applications in such areas as biomedical apparatus [2], detector system [3], LCD back lighting [4], general decorative illuminations, etc. LEDs are direct band-gap semiconductor $\mathrm{p}-\mathrm{n}$ junction diodes. The band-gap of the semiconductor, controlled by mixing different proportion of III-V elements from the periodic table, defines the light color frequency of the diode [5]. The band-gap of the LED may therefore change from time to time during operation where the junction temperature changes with power dissipation and the ambient temperature as well as device aging. Such properties impose engineering challenges in correcting color drift due to device, temperature and aging variations.

We consider the LED color control problem as a system control problem in which we aim to reduce the complexity of the control-to-output path. The non-linearity of the forward driving current to LED light and color output has been reduced dramatically using PWM control of the fixed forward current drive, resulting in a one-step calibration of the device variation compensation at maximum light intensity output. Lacking a

This work is supported by Hong Kong Research Grant Council under a competitive earmarked research grant (PolyU 5306/06E). proper method of measuring the junction temperature of the LED, a feedback system using RGB sensors has been proposed to keep track of the output light intensity for correcting the otherwise impossible to measure temperature variation [6]. However, as the feedback control uses a light intensity sensor whose value varies as the LED dims down, measuring errors can be quite large when the intensity gets small. Further, current technology using $\mathrm{A} / \mathrm{D}$ converters which has a higher fractional change for less number of voltage representation makes the system less accurate for higher dimming factor. The feedback control system cannot correct the aging effect of devices as the light sensors themselves are prone to aging variation. Regular frequent calibrations are still needed for such feedback schemes. In this paper we propose a much simpler and more practical technique for color control by measuring the optical and electrical characteristics of the LED during the turn-on duration of PWM forward driving current. The forward-voltage-to-junction-temperature variation is roughly linear under fixed driving current. This property has been widely exploited for sensing the ambient temperature. Thus, temperature compensation can be achieved by detecting instantly the diode forward voltage.

This paper is organized as follows. Section II gives an overview of the RGB LED color lighting system. LED diode junction temperature compensation technique is given in Section III. Implementation details of the system are given in Section IV. The performance of the proposed compensation technique is evaluated in Section V. Section VI concludes this paper.

\section{Overview of RGB LED COLOR CONTROL}

The data points on the CIE 1976 chromaticity diagram shown in Fig. 1 gives the color coordinates $\left(u^{\prime}, v^{\prime}\right)$ of typical red, green and blue LEDs having wavelengths from $380 \mathrm{~nm}$ to $700 \mathrm{~nm}$ for a step size of $50 \mathrm{~nm}$. The diagram was made in a way to give an even color perceptible difference for the distance of two color points independent of the absolute positions of the color points. A color distance of $\delta u^{\prime} v^{\prime}<0.002$ is indistinguishable to humans. Errors within this small distance are considered very acceptable for most applications. Mixing the light intensity of the three LEDs having wavelengths of 


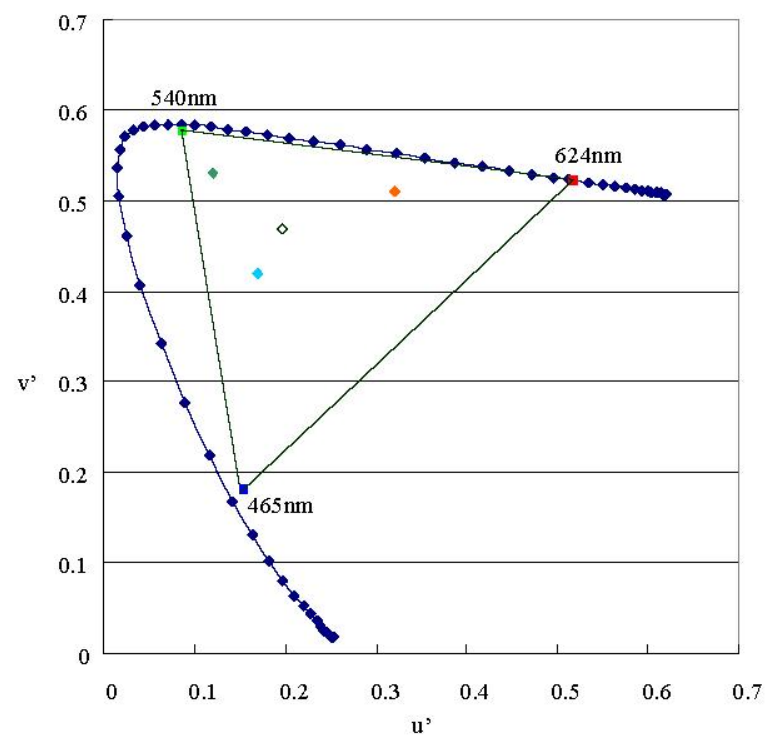

Fig. 1. Color coordinates of typical LED of center wavelengths ranging from $700 \mathrm{~nm}$ to $380 \mathrm{~nm}$. Adjacent dots are separated by $50 \mathrm{~nm}$ in center wavelength. LEDs with center wavelengths of $620 \mathrm{~nm}$ (red), $540 \mathrm{~nm}$ (green) and $465 \mathrm{~nm}$ (blue) can generate color points within the triangle shown in this figure.

$620 \mathrm{~nm}, 540 \mathrm{~nm}$ and $465 \mathrm{~nm}$ can produce all colors within the triangle whose comers are at the color coordinates of the RGB LEDs. The color accuracy of light mixed by the RGB LEDs is therefore dependent on

1) the stability of the color points of the RGB LEDs; and

2) the accuracy of LED light dimming method.

We assume that the light stability of color points of LEDs is largely dependent on the junction temperatures $T_{R}, T_{G}$ and $T_{B}$ of the red, green, and blue diodes. The effect of component aging is ignored here as it is a very slow process and can be easily compensated by regular calibrations.

An intended light output is usually expressed in terms of the so called tristimulus, denoted by $\mathbf{X}=(X Y Z)^{t}$, and also the dimming factor, denoted by $\mathrm{d}=\left(d_{r} d_{g} d_{b}\right)^{t}$ and given by

$$
\mathbf{d}=\mathbf{f}_{\mathrm{T}}(\mathbf{X}),
$$

where $f_{T}$ is a column vector function whose parameters change with $\mathbf{T}$, which is a column vector of the LED junction temperatures, i.e., $\mathbf{T}=\left(T_{R} T_{G} T_{B}\right)^{t}$. Note that $\mathbf{T}$ depends on both $\mathrm{d}$ and the ambient temperature.

Without precise information of temperature change inside the LED diode junction, the control of light output from (1) can only be achieved by a feedback loop that senses the light outputs from the RGB LEDs and adjusts the control parameter $\mathbf{d}$ to compensate for the light output drifts as defined from $\mathbf{f}_{\mathrm{T}}$ [6]. Indirect temperature compensation techniques like sensing the temperature of the heat sink on which the LEDs are mounted [7] or the method of two-diode driving voltages [3] may introduce too much error in estimating diode junction temperature and increase system complexity. Thus, light sensing is still important for ensuring high performance.

\section{TECHNIQUE OF JUNCTION TEMPERATURE}

\section{COMPENSATION AND EXPERIMENTAL MEASUREMENTS}

Equation (1) can be highly nonlinear if inappropriate control method and/or working color range are used. It is widely known that dimming using PWM is preferred over the use of amplitude modulation because of the the linear relationship between the duty cycle $\mathrm{d}$ and the LED color light output. We have confirmed this by experimental measurement, as shown in Figs. 2, 3, 4. The tristimulus $\mathbf{X}=\left(\begin{array}{lll}X & Y & Z\end{array}\right)^{t}$ changes linearly with the junction temperature and hence also with the A/D converted values of RGB diode voltages when drivers operate at a stable duty cycle. Also, the tristimulus $\mathbf{X}$ changes linearly with the duty cycle at a stable junction temperature. Small measurement errors can be compensated by calibration. So, we can simply measure the above linear correspondence at one duty cycle value, such as unity, and derive the corresponding tristimulus at the other duty cycle values accordingly. The linearity of the dependence of RGB lights on the RGB diode voltages makes programming very simple. Moreover, the curves (purple dashed curves) measured at a given ambient temperature and different duty cycles, as shown in Fig. 2, show that the effect of junction temperature drift caused by power dissipation and junction temperature compensation is very significant, as illustrated by the purple dashed curve in Fig. 2.

We use the Lamina BL-4000 RGB LED light engine to illustrate how essential parameters contained in $\mathbf{f}_{\mathrm{T}}$ of (1) can be found. A constant current of $330 \mathrm{~mA}$ during the turn-on duration of the PWM cycle is applied to all the red, green and blue LEDs at ambient temperature. We have measured $\mathbf{X}_{o r}$, $\mathbf{X}_{o g}$, and $\mathbf{X}_{o b}$ versus diode forward voltages of the LEDs, i.e., $V_{d r}, V_{d g}$ and $V_{d b}$, at $\left(d_{r} d_{g} d_{b}\right)=(1,1,1)$. We use the LED heat sink temperature as the variation parameter.

The mixed color is therefore given as

$$
\left(\begin{array}{c}
X_{o} \\
Y_{o} \\
Z_{o}
\end{array}\right)=d_{r}\left(\begin{array}{c}
X_{o r} \\
Y_{o r} \\
Z_{o r}
\end{array}\right)+d_{g}\left(\begin{array}{c}
X_{o g} \\
Y_{o g} \\
Z_{o g}
\end{array}\right)+d_{b}\left(\begin{array}{c}
X_{o b} \\
Y_{o b} \\
Z_{o b}
\end{array}\right)
$$

where

$$
\left(\begin{array}{c}
X_{o i} \\
Y_{o i} \\
Z_{o i}
\end{array}\right)=\left(\begin{array}{c}
\alpha_{i 1} V_{d i}+\alpha_{i 2} \\
\alpha_{i 3} V_{d i}+\alpha_{i 4} \\
\alpha_{i 5} V_{d i}+\alpha_{i 6}
\end{array}\right)
$$

with $i=r, g, b$ and $\alpha_{i j}$ being coefficients of least square fits.

Since $\mathbf{X}_{o r}, \mathbf{X}_{o g}$, and $\mathbf{X}_{o b}$ are linearly independent, it is always possible to determine a unique $\mathbf{d}$ such that (2) is satisfied. If $\mathbf{X}_{o r}, \mathbf{X}_{o g}$, and $\mathbf{X}_{o b}$ are temperature invariant and $\mathrm{d}$ is well controlled, the light color output will be very stable as given in (2). However, the three color bases $\mathbf{X}_{o r}, \mathbf{X}_{o g}$, 

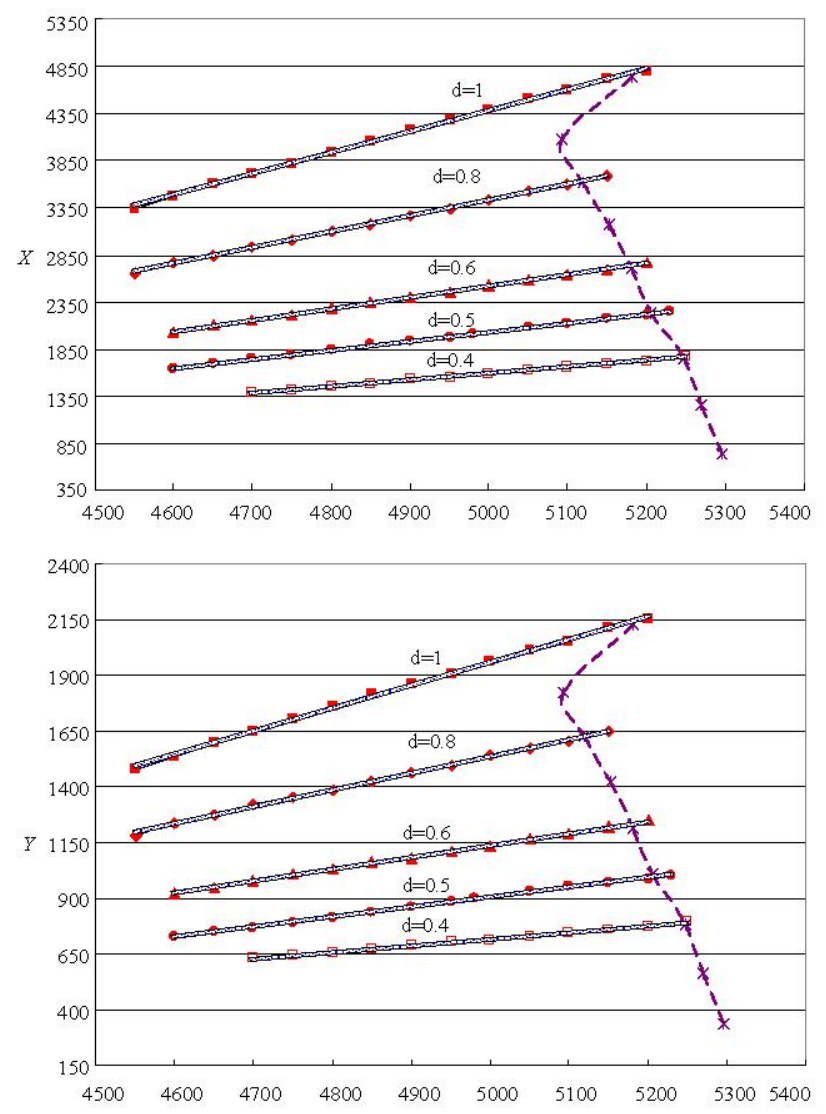

Fig. 2. Experimental red channel light tristimulus $\mathbf{X}$ versus diode voltage (digital) of red light at different duty cycles. Diamonds are data points and lines are fitted with the data points. Purple dashed curve is measured at room ambient temperature $\left(25^{\circ} \mathrm{C}\right)$ with different duty cycles. Note that $Z$ in $\mathbf{X}$ of red light is almost zero and not shown in this figure.

and $\mathbf{X}_{o b}$ are temperature dependent, as given in (3). Using (2) and (3), the desired color can be achieved. The transformation of $(X, Y, Z)$ to other CIE color space systems is monotonic. For instance, the relation between $X Y Z$ (tristimulus) and $u^{\prime} v^{\prime}$ (chromaticity) is simply

$$
\begin{aligned}
& u^{\prime}=\frac{4 X}{X+15 Y+3 Z} \\
& v^{\prime}=\frac{9 X}{X+15 Y+3 Z}
\end{aligned}
$$

The whole process can be done automatically with the help of a well calibrated visible light spectrometer.

\section{IMPLEMENTATION OF RGB LED COLOR CONTROL SYSTEM}

The Lamina BL-4000 RGB LED light engine is selected for detailed study and illustration. Each red, green and blue light channel is driven separately by a current-controlled switching converter, as shown in Fig. 5. The switching converter behaves essentially as a current source whose magnitude is controlled by a PWM signal. Our converter is modified from a control IC LT1510 designed for battery chargers operating at a switching
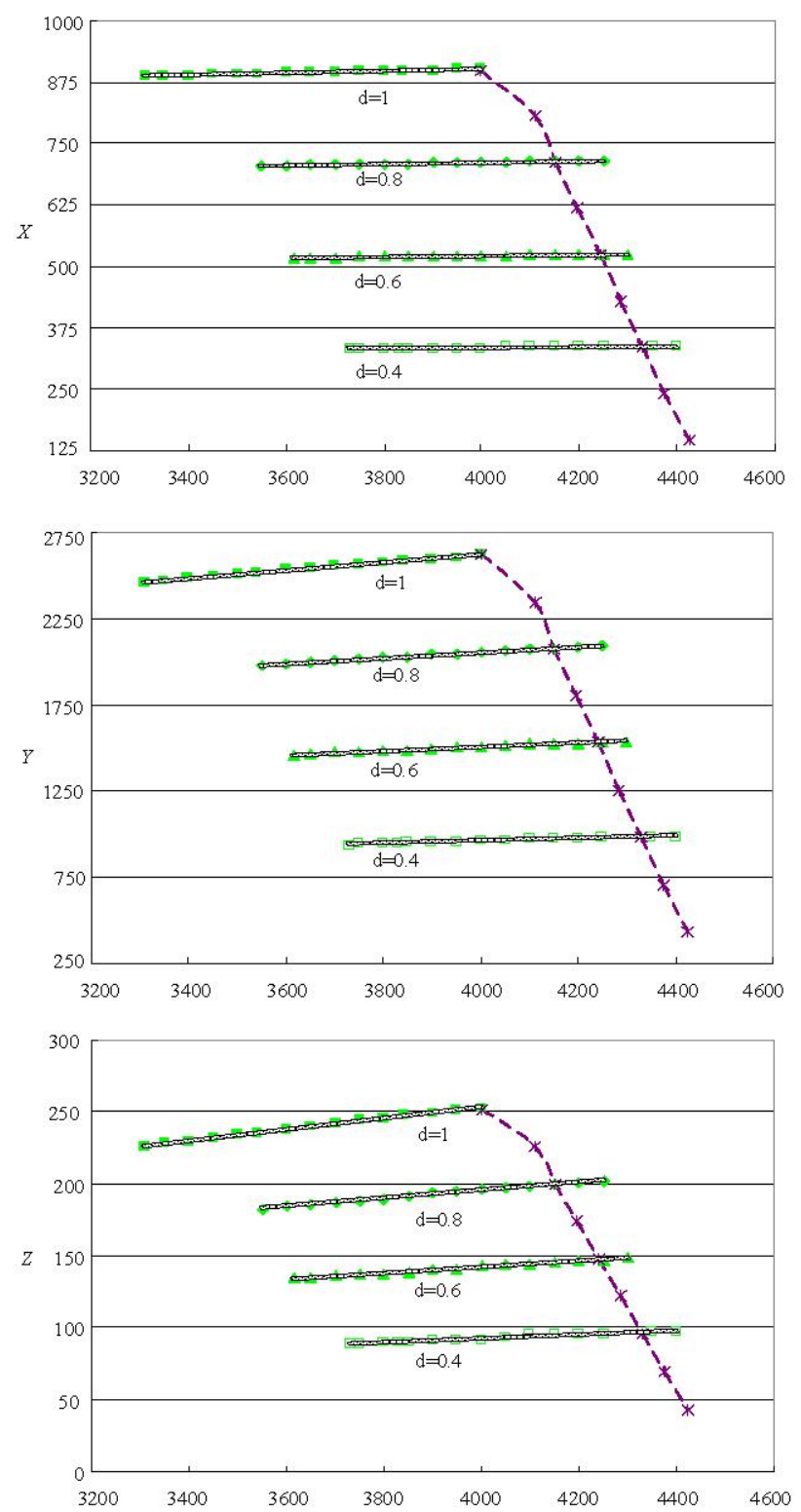

Fig. 3. Experimental green channel light tristimulus $\mathbf{X}$ versus diode voltage (digital) of green light at different duty cycles with the same condition as in Fig. 2. Purple dashed curve is measured at room ambient temperature $\left(25^{\circ} \mathrm{C}\right)$ with different duty cycles.

frequency of $200 \mathrm{kHz}$. Component values have been optimized for fast transient of the output current under a PWM gate pulse, $V_{\mathrm{PWM}}$, which is applied at a frequency of $200 \mathrm{~Hz}$ to the MOS transistor, as shown in Fig. 5.

Three identical circuits, each resembling Fig. 5, are used for driving the red, green and blue LED channels, the schematic of which is shown in Fig. 6. The LED drivers are controlled by the microcontroller PIC18C1320. Fig. 7 shows a photo of the LED with the microcontroller and power converters. Fig. 8 shows typical waveforms of the forward voltage across the red, green and blue diodes under constant current at $330 \mathrm{~mA}$ which 

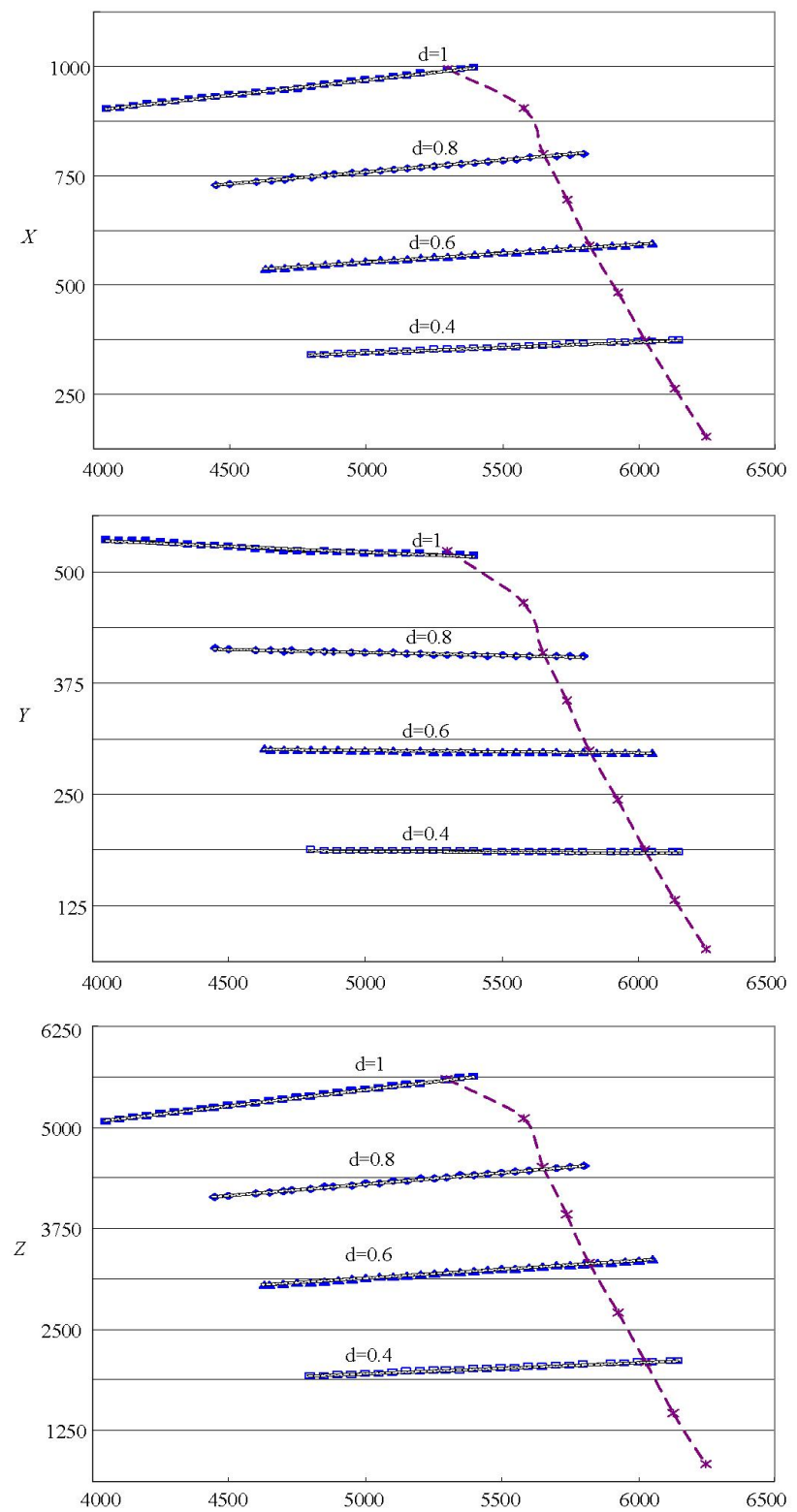

Fig. 4. Experimental blue channel light tristimulus $\mathbf{X}$ versus diode voltage (digital) of blue light at different duty cycles with same condition as in Fig. 2. Purple dashed curve is measured at room ambient temperature $\left(25^{\circ} \mathrm{C}\right)$ with different duty cycles.

is pulse-width modulated for brightness control. Then, the voltage during the turn-on duration is detected and converted to digital format suitable for PIC18C1320 programming.

During the calibration phase, digital format of the diode turn-on voltages is sent from the PIC18C1320 to a personal computer via RS232 connection at a rate of 200 samples per second. The digital temperature $V_{d i}(i=r, g, b)$ is smoothened using a simple integrator formula, i.e.,

$$
V_{d i, \text { current }}=\alpha V_{d i \text {,measured }}+(1-\alpha) V_{d i, \text { previous }}
$$

where the current value of the digital temperature $V_{d i \text {,current }}$

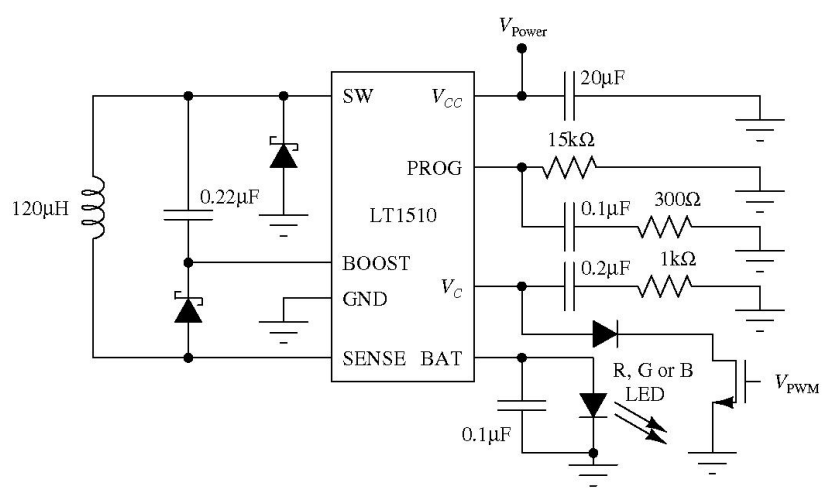

Fig. 5. Switching converter with PWM control as constant current driver.

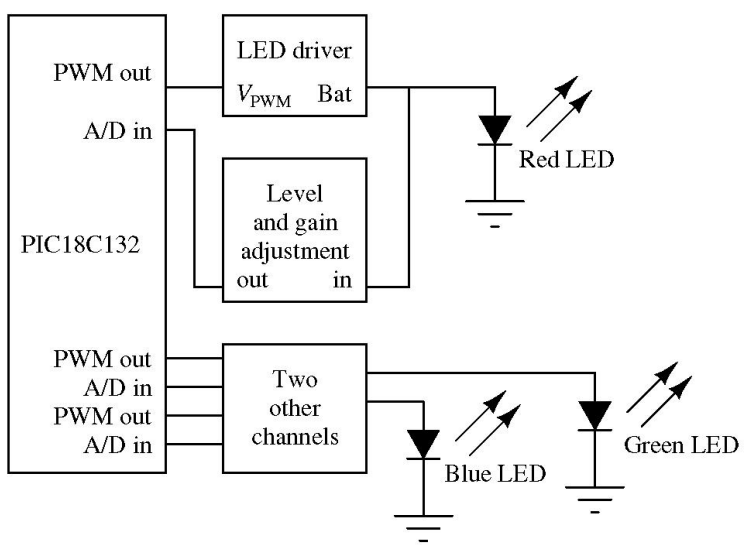

Fig. 6. Schematic of the RGB LED color control system.

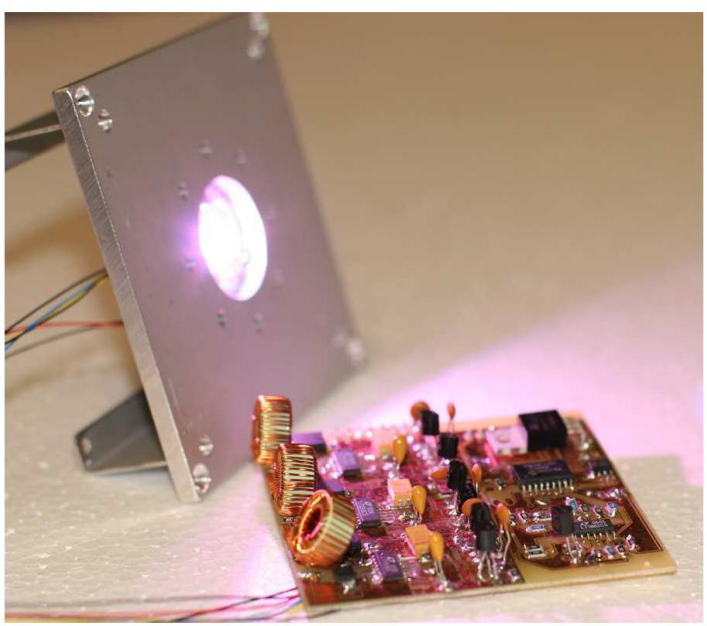

Fig. 7. LED lamp with driver and control circuit.

is taken as the weighted value of the actually measured one

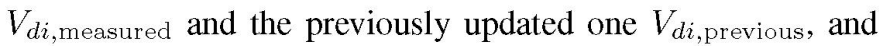
$\alpha$ is essentially an integration constant which is adjusted to smoothen the averaged waveform. Here, we use $\alpha=0.05$. Then, $V_{d i}$ is compared graphically on the computer display with the measured diode voltages, as shown in Fig. 9. 


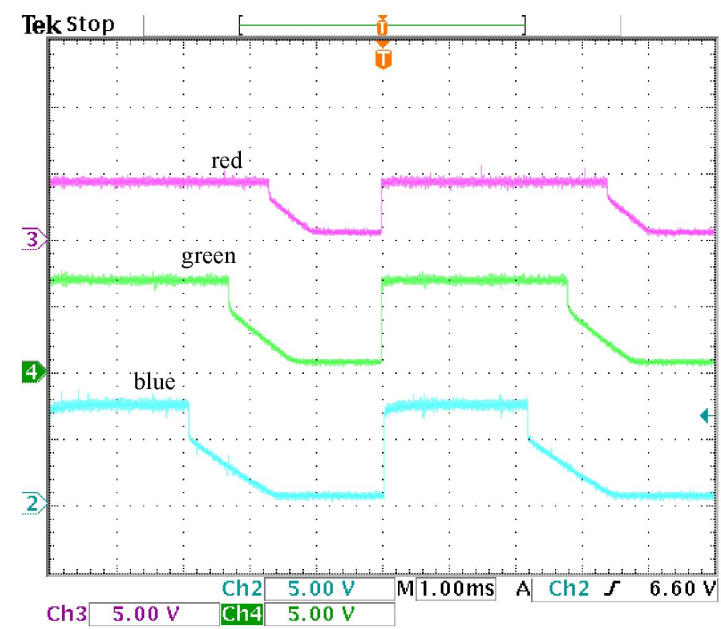

Fig. 8. LED diode forward voltage waveforms; upper trace for red LED, middle trace for green LED and lower trace for blue LED

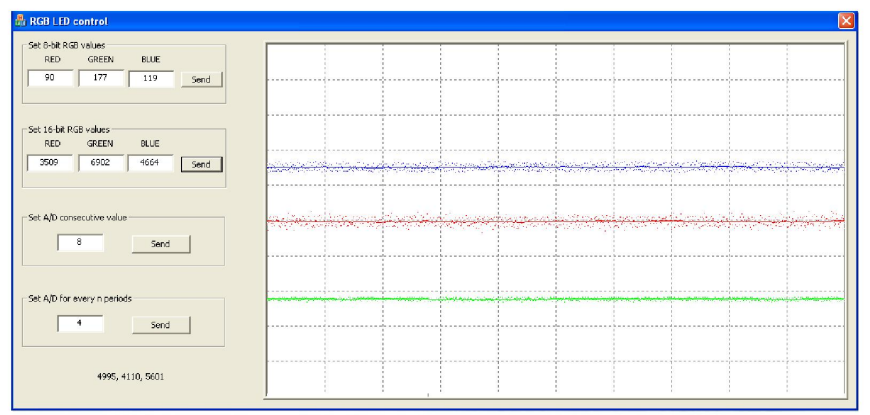

Fig. 9. Comparison of point samples of digital temperature $V_{d i}$ with averaged waveforms using (6) for $i=r, g, b$.

The computer is also connected to a spectroradiometric system from Oceanoptics Inc. to measure the absolute or relative irradiance of LED lights [8] through a USB local bus connection. Then, SpectraSuite Software calculates tristimulus $X Y Z$, hue $u^{\prime} v^{\prime} w^{\prime}$, and other correlated color parameters from the spectrum distribution. The user interface panel is shown in Fig. 10. Note that the spectroradiometric system can be easily programmed to automate the measurements.

The 18 unknown coefficients in (3) can be calculated from the data obtained from this computer controlled system. The algorithm is as follows. For each duty cycle value equal to $1,0.8,0.6$ and 0.4 , we measure the tristimulus $X Y Z$ corresponding to the digital diode forward voltage while the LED heat sink is heated up from room temperature to about $100^{\circ} \mathrm{C}$. The measurement is done for each of the red, green, blue LEDs. Results are shown earlier in Figs. 2, 3, 4.

By curve-fitting in Figs. 2, 3, 4, the coefficients can be obtained as

$$
\left(\begin{array}{c}
X_{o r} \\
Y_{o r} \\
Z_{o r}
\end{array}\right)=\left(\begin{array}{c}
2.2421 * V_{d r}-6832.1 \\
1.0281 * V_{d r}-3183.5 \\
0.0 * V_{d r}-0.0
\end{array}\right)
$$

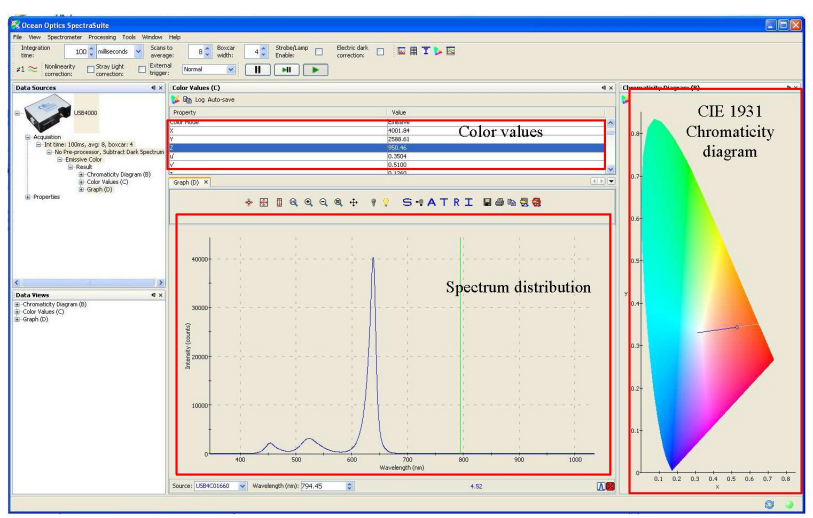

Fig. 10. SpectraSuite Software interface with several function blocks.

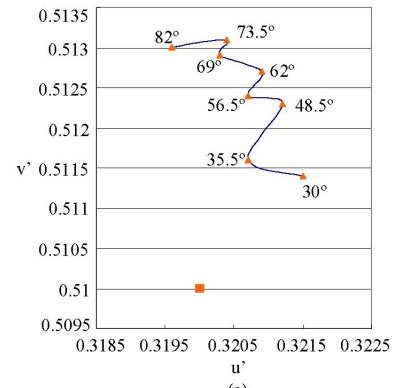

(a)
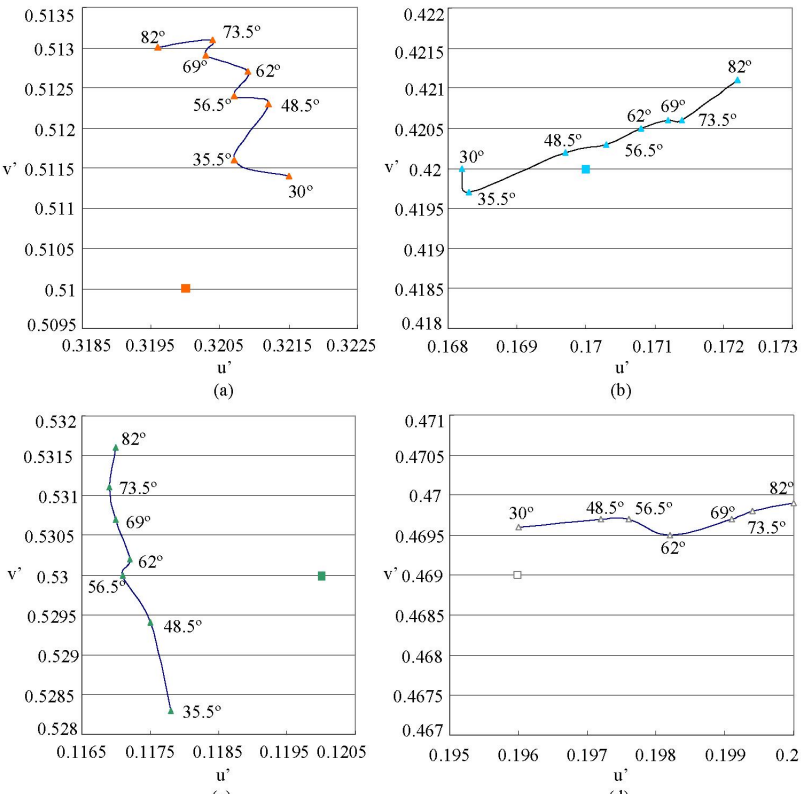

(b)

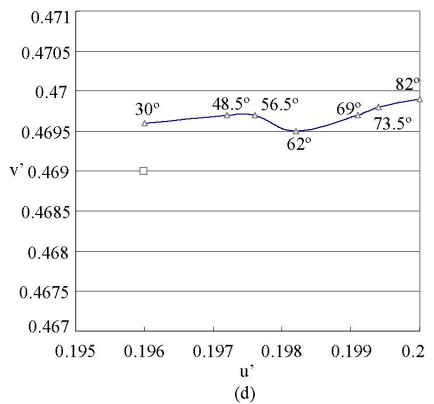

Fig. 11. Evaluation of output color points, marked with small triangles, changing with different heat sink temperatures for set-point colors at (a) light red (b) light blue (c) light green and (d) D65 white, marked with small squares.

$$
\begin{gathered}
\left(\begin{array}{c}
X_{o g} \\
Y_{o g} \\
Z_{o g}
\end{array}\right)=\left(\begin{array}{c}
0.020625 * V_{d g}+818.925 \\
0.232025 * V_{d g}+1695.625 \\
0.039175 * V_{d g}+96.865
\end{array}\right) \\
\left(\begin{array}{c}
X_{o b} \\
Y_{o b} \\
Z_{o b}
\end{array}\right)=\left(\begin{array}{c}
0.07005 * V_{d b}+618.8 \\
-0.01385 * V_{d b}+590.725 \\
0.406475 * V_{d b}+3429.75
\end{array}\right)
\end{gathered}
$$

These coefficients are then used for a test run of color accuracy in the computer. If the color accuracy is within tolerance, they will be programmed into the PIC18C132 for stand-alone realtime control of light colors.

\section{Evaluation}

Four color points are chosen to evaluate the performance of the proposed method. We examine the color and brightness 
change with temperature at four different points on the color coordinates, namely D65 white, light red, light green and light green, with corresponding CIE 1976 chromaticity coordinates at $\left(u^{\prime}, v^{\prime}\right)=(0.196,0.469),(0.32,0.51),(0.25,0.50)$ and $(0.17,0.42)$, respectively, as shown in Fig. 1 . For about $50^{\circ} \mathrm{C}$ change in temperature of the heat sink, the position of color point on the CIE $1976 u^{\prime} v^{\prime}$ plane and the brighness in $Y$ value are plotted in Fig. 11 and Fig. 12 respectively. The maximum color difference is 0.0041 which has been recorded for D65 white, as shown in Fig. 13. The measurement also employs the afore-described spectroradiometric setup under the same condition so that the experimental results are consistent and reliable. The error is mainly due to the precision of the duty cycle and can be made very small by using a high bit resolution timer of the micro-controller. Users can simply input the desired color tristimulus values and then the system can automatically adjust the duty cycles of RGB LED drivers for stable color light intensity output without the use of any other devices such as sensors.

\section{CONCLUSION}

Color control is an important issue in the design and manufacture of LED lighting systems. Due to device variation, aging and sensing nonlinearity, achieving color precision and standardization for large number of LEDs is often a difficult task. A temperature compensation technique has been proposed in this paper. The implementation technique is outlined and verified by some experimental data. The technique can control the color effectively, and can also dramatically reduce the complexity and eliminate the need for using expensive feedback systems involving light sensors. The system can be readily ported to a fully automatic computerized system for industrial implementation.

\section{REFERENCES}

[1] F. Richard, "Light-emitting diodes: a guide to the technology and its implications," Bracknell: BSRIA, 2005.

[2] A. E. Moe, N. Banani, L. A. Lee, B. Marquardt, and D. M. Wilson, "Enhanced fluorescence emission using a programmable, reconfigurable LED-array based light source," Proc. Int. Conf. Engineering in Medicine and Biology Society, vol. 3, pp. 2090-2093, Sept. 2004.

[3] K. Saucke, G. Pausch, J. Stein, H.-G. Ortlepp, and P. Schotanus, "Stabilizing scintillation detector systems with pulsed LEDs: a method to derive the LED temperature from pulse height spectra," IEEE Trans. Nuclear Science, vol. 52, no. 6, pp. 3160-3165, Dec. 2005

[4] C. C. Chen, C-Y. Wu, and T-F. Wu, "LED back-light driving system for LCD panels," IEEE Applied Power Electronics Conf. Exposition, pp. 381-385, March 2006.

[5] D. A. Steigerwald, J. C. Bhat, D. Collins, R. M. Fletcher, M. O. Holcomb, and M. J. Ludowise, "Ilumination with solid state lighting technology," IEEE J. Selected Topics in Quantum Electronics, vol. 8, no. 2, pp. 310-320, March/April 2002

[6] S. Muthu, F. J. Schuurmans, and M. D. Pashley, "Red, green, and blue LED based white light generation: issues and control" Proc. Industry Applications Conf., pp. 327-333, Oct. 2002.

[7] S. Muthu and J. Gaines, "Red, green, and blue LED-based white light source: implementation challenges and control design," Proc. Industry Applications Conf., pp. 515-522, Oct. 2003.

[8] Oceanoptics Inc. Website[Online]: http://www.oceanoptics.com/products/ spectroradiometric.asp.
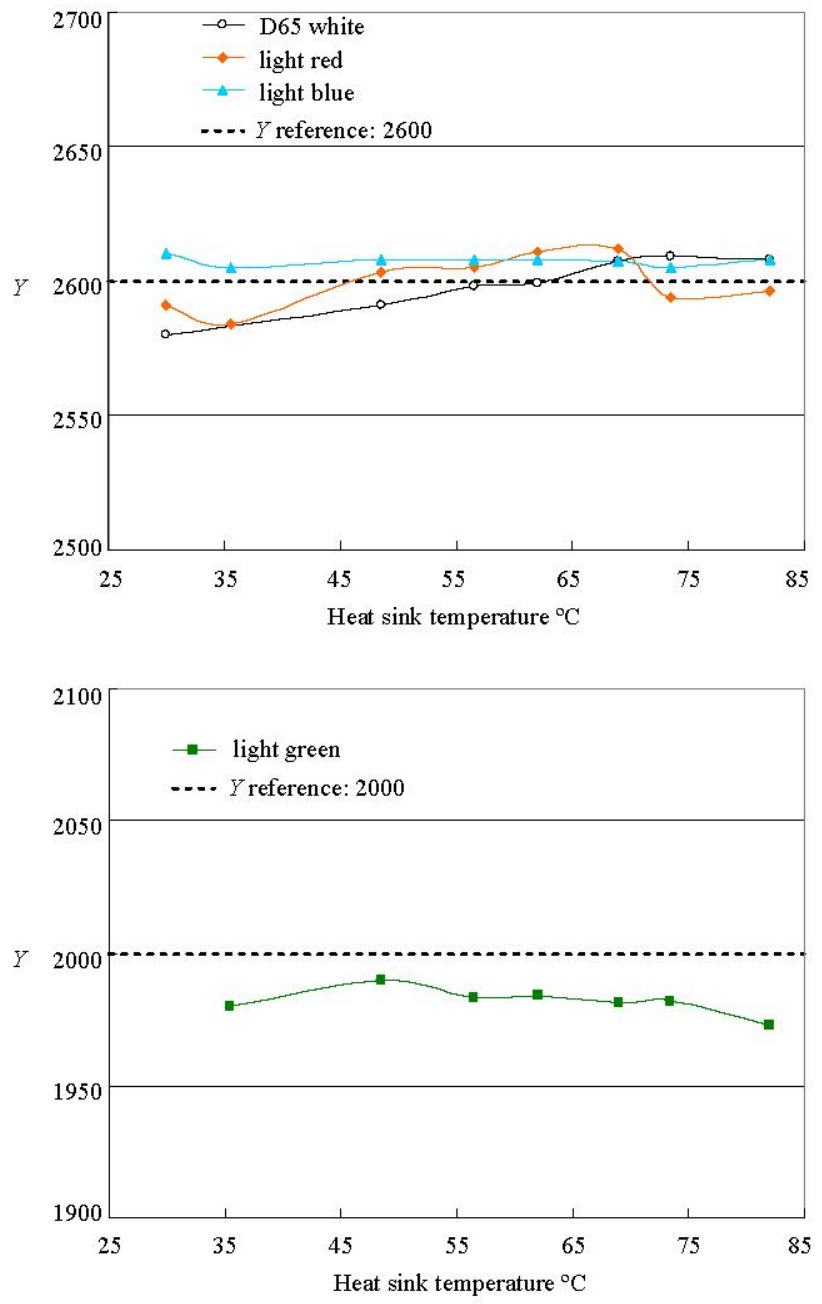

Fig. 12. Evaluation of output brightness difference in $Y$ value, corresponding to Fig. 11. The $Y$ reference for D65 white, light blue and light red is $Y=$ 2600 . The $Y$ reference for light green is $Y=2000$.

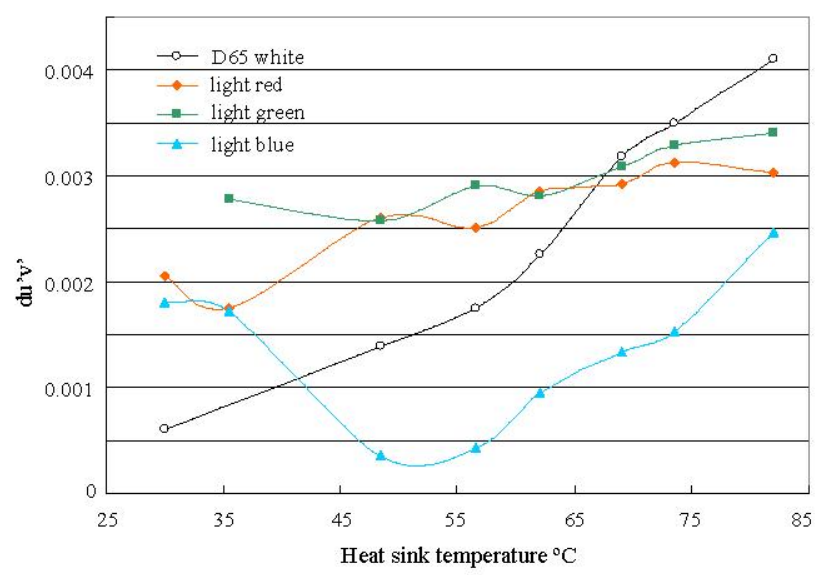

Fig. 13. Evaluation of output color difference using $\delta u^{\prime} v^{\prime}=$ $\sqrt{\left(\delta u^{\prime}\right)^{2}+\left(\delta v^{\prime}\right)^{2}}$, corresponding to Fig. 11 . 